\title{
SOME FIXED POINT THEOREMS ON FUZZY METRIC SPACES WITH IMPLICIT RELATIONS
}

\author{
Ishak Altun and Duran Turkoglu
}

Abstract. In this paper, we give some fixed point theorems on fuzzy metric spaces with an implicit relation. Our results extend and generalize some fixed point theorems on complete fuzzy metric spaces by using a new technique.

\section{Introduction and preliminaries}

The concept of fuzzy sets was introduced by Zadeh [25]. It was developed extensively by many authors and used in various fields. To use this concept in topology and analysis, several researchers have defined fuzzy metric spaces in various ways. Many authors have studied fixed point theory in fuzzy metric spaces for fuzzy mappings (see [1]-[6], [11], [13]-[16], [21], [22]).

In this paper, we give an implicit relation on fuzzy metric spaces and present some fixed point theorems. This theorems are generalization of some previous fixed point theorems given by some authors.

Now, we begin with some definitions.

Definition 1 ([20]). A binary operation $\star:[0,1] \times[0,1] \rightarrow[0,1]$ is called a continuous $t$-norm if $([0,1], \star)$ is an Abelian topological monoid with the unit 1 such that $a \star b \leq c \star d$ whenever $a \leq c$ and $b \leq d$ for all $a, b, c, d \in[0,1]$.

Examples of $t$-norms are $a \star b=a b$ and $a \star b=\min \{a, b\}$.

Definition 2 ([10]). The 3 -tuple $(X, M, \star)$ is called a fuzzy metric space if $X$ is an arbitrary set, $\star$ is a continuous $t$-norm and $M$ is a fuzzy set in $X^{2} \times(0, \infty)$ satisfying the following conditions:

$(\mathrm{Fm}-1) \quad M(x, y, t)>0$,

(Fm-2) $M(x, y, t)=1$ if and only if $x=y$,

(Fm-3) $M(x, y, t)=M(y, x, t)$,

$(\mathrm{Fm}-4) M(x, y, t) \star M(y, z, s) \leq M(x, z, t+s)$,

$(\mathrm{Fm}-5) M(x, y, \cdot):(0, \infty) \rightarrow[0,1]$ is continuous for all $x, y, z \in X$ and $t, s>0$.

Received November 8, 2006.

2000 Mathematics Subject Classification. Primary 54H25; Secondary 54A40.

Key words and phrases. fuzzy metric space, common fixed point, compatible mappings, implicit relation. 
In the following example, we show that every metric induces a fuzzy metric.

Example $1([10])$. Let $(X, d)$ be a metric space. Define $a \star b=a b$ (or $a \star b=$ $\min \{a, b\})$ and, for all $x, y \in X$,

$$
M(x, y, t)=\frac{t}{t+d(x, y)} .
$$

Then $(X, M, \star)$ is a fuzzy metric space. We call this fuzzy metric $M$ induced by the metric $d$ the standard fuzzy metric.

Lemma 1 ([11]). For all $x, y \in X, M(x, y, \cdot)$ is nondecreasing on $(0, \infty)$.

Proof. Suppose that $M(x, y, s)<M(x, y, t)$ for some $0<t<s$. Then we have

$$
M(x, y, t) \star M(y, y, s-t) \leq M(x, y, s)<M(x, y, t) .
$$

By $(\mathrm{Fm}-2), M(y, y, s-t)=1$ and thus $M(x, y, t) \leq M(x, y, s)<M(x, y, t)$, which is a contradiction.

Definition $3([24])$. Let $(X, M, \star)$ be a fuzzy metric space.

(1) A sequence $\left\{x_{n}\right\}$ in $X$ is called a Cauchy sequence if, for any $0<\varepsilon<1$ and $t>0$, there exists $n_{0} \in \mathbb{N}$ such that $M\left(x_{n}, x_{m}, t\right)>1-\varepsilon$ for all $m, n \geq n_{0}$.

(2) A sequence $\left\{x_{n}\right\}$ in $X$ is said to be convergent to a point $x \in X$ if, for any $0<\varepsilon<1$ and $t>0$, there exists $n_{0} \in \mathbb{N}$ such that $M\left(x_{n}, x, t\right)>$ $1-\varepsilon$ for all $n \geq n_{0}$.

(3) A fuzzy metric space $(X, M, \star)$ in which every Cauchy sequence is convergent is said to be complete.

Lemma $2([16])$. Let $(X, M, \star)$ be a fuzzy metric space such that, for all $x, y \in$ $X, M(x, y, t) \rightarrow 1$ as $t \rightarrow \infty$. If there exists a constant $0<k<1$ such that, for all $t>0$,

$$
M(x, y, k t) \geq M(x, y, t)
$$

for all $x, y \in X$, then $x=y$.

Proof. If $M(x, y, k t) \geq M(x, y, t)$ for all $t>0$ and some constant $0<k<1$, then we have

$$
M(x, y, s) \geq M\left(x, y, \frac{s}{k}\right) \geq M\left(x, y, \frac{s}{k^{2}}\right) \geq \cdots \geq M\left(x, y, \frac{s}{k^{n}}\right)
$$

for all $s>0$ and $x, y \in X$. Letting $n \rightarrow \infty$, we have $M(x, y, s)=1$ and thus $x=y$.

Now, we give some definitions of compatible mappings, some properties and an example in fuzzy metric spaces.

Definition 4 ([16]). Let $A$ and $B$ be mappings from a fuzzy metric space $(X, M, \star)$ into itself. The mappings $A$ and $B$ are said to be compatible if, for all $t>0, M\left(A B x_{n}, B A x_{n}, t\right) \rightarrow 1$ as $n \rightarrow \infty$ whenever $\left\{x_{n}\right\}$ is a sequence in $X$ such that $A x_{n}, B x_{n} \rightarrow z$ as $n \rightarrow \infty$ for some $z \in X$. 
Definition 5 ([7]). Let $A$ and $B$ be mappings from a fuzzy metric space $(X, M, \star)$ into itself. The mappings $A$ and $B$ are said to be compatible of type $(\alpha)$ if, for all $t>0, M\left(A B x_{n}, B B x_{n}, t\right) \rightarrow 1$ and $M\left(A A x_{n}, B A x_{n}, t\right) \rightarrow 1$ as $n \rightarrow \infty$ whenever $\left\{x_{n}\right\}$ is a sequence in $X$ such that $A x_{n}, B x_{n} \rightarrow z$ as $n \rightarrow \infty$ for some $z \in X$.

Definition 6 ([8]). Let $A$ and $B$ be mappings from a fuzzy metric space $(X, M, \star)$ into itself. The mappings $A$ and $B$ are said to be compatible of type $(\beta)$ if, for all $t>0, M\left(A A x_{n}, B B x_{n}, t\right) \rightarrow 1$ as $n \rightarrow \infty$ whenever $\left\{x_{n}\right\}$ is a sequence in $X$ such that $A x_{n}, B x_{n} \rightarrow z$ as $n \rightarrow \infty$ for some $z \in X$.

Proposition $1([7])$. Let $(X, M, \star)$ be a $F M$-space with $t \star t \geq t$ for all $t \in[0,1]$ and $A, B$ be continuous mappings from $X$ into itself. Then $A$ and $B$ are compatible if and only if they are compatible of type $(\alpha)$.

Proposition $2([8])$. Let $(X, M, \star)$ be a $F M$-space with $t \star t \geq t$ for all $t \in[0,1]$ and $A, B$ be continuous mappings from $X$ into itself. Then $A$ and $B$ are compatible if and only if they are compatible of type $(\beta)$.

Proposition $3([8])$. Let $(X, M, \star)$ be a $F M$-space with $t \star t \geq t$ for all $t \in[0,1]$ and $A, B$ be continuous mappings from $X$ into itself. Then $A$ and $B$ are compatible of type $(\alpha)$ if and only if they are compatible of type $(\beta)$.

Proposition $4([22])$. Let $(X, M, \star)$ be a FM-space with the $t \star t \geq t$ for all $t \in[0,1]$. A and $S$ be compatible maps of type $(\beta)$ from $X$ into itself and let $\left\{x_{n}\right\}$ be a sequence in $X$ such that $A x_{n}, S x_{n} \rightarrow z$ for some $z \in X$. Then we have the following

(I) $S S x_{n} \rightarrow A z$ if $A$ is continuous at $z$,

(II) $A A x_{n} \rightarrow S z$ if $S$ is continuous at $z$,

(III) $A S z=S A z$ and $A z=S z$ if $A$ and $S$ are continuous at $z$.

Example 2. Let $X=[0, \infty)$ with the metric $d$ defined by $d(x, y)=|x-y|$. For each $t \in(0, \infty)$ and $x, y \in X$, define $M$ by

$$
M(x, y, t)=\left[\exp \left(\frac{|x-y|}{t}\right)\right]^{-1} .
$$

Clearly, $(X, M, \star)$ is a $F M$-space, where $\star$ is define by $a \star b=\min \{a, b\}$. Let $A$ and $B$ be defined by $A x=1$ for all $x \in[0,1], A x=1+x$ for all $x \in(1, \infty)$ and $B x=1+x$ for all $x \in[0,1), B x=1$ for all $x \in[1, \infty)$. Let $\left\{x_{n}\right\}$ be a sequence in $X$ such that $\lim _{n \rightarrow \infty} A x_{n}=\lim _{n \rightarrow \infty} B x_{n}=z$. By definition of $A$ and $B$, $z \in\{1\}$ and $\lim _{n \rightarrow \infty} x_{n}=0$. $A$ and $B$ both are discontinuous at $z=1$. Therefore, we have

$$
M\left(A A x_{n}, B A x_{n}, t\right) \rightarrow 1, M\left(A A x_{n}, B B x_{n}, t\right) \rightarrow 1 \text { as } n \rightarrow \infty
$$


Also, we consider the sequence $\left\{x_{n}\right\}$ in $X$ defined by $x_{n}=\frac{1}{2 n}, n=1,2, \ldots$. Then we have $A x_{n}, B x_{n} \rightarrow 1$ as $n \rightarrow \infty$. Further, for $t>0$, we have

$$
\begin{aligned}
M\left(A B x_{n}, B A x_{n}, t\right) & \rightarrow\left[\exp \left(\frac{1}{t}\right)\right]^{-1}<1, \\
M\left(A B x_{n}, B B x_{n}, t\right) & \rightarrow\left[\exp \left(\frac{1}{t}\right)\right]^{-1}<1
\end{aligned}
$$

as $n \rightarrow \infty$. Therefore, $A, B$ is compatible of type $(\beta)$, but they are neither compatible nor compatible of type $(\alpha)$.

\section{Implicit relation}

Implicit relations on metric spaces have been used in many articles (see [13], [19]-[17], [23]).

Let $I=[0,1], \star$ be a continuous $t$-norm and $F: I^{6} \rightarrow \mathbb{R}$ be a continuous function. Now, we consider the following conditions:

(F-1) $F$ is nonincreasing in the fifth and sixth variables,

(F-2) if, for some constant $k \in(0,1)$, we have

$$
F\left(u(k t), v(t), v(t), u(t), 1, u\left(\frac{t}{2}\right) \star v\left(\frac{t}{2}\right)\right) \geq 1
$$

or

$$
F\left(u(k t), v(t), u(t), v(t), u\left(\frac{t}{2}\right) \star v\left(\frac{t}{2}\right), 1\right) \geq 1
$$

for any fixed $t>0$ and any nondecreasing functions $u, v:(0, \infty) \rightarrow I$ with $0<u(t), v(t) \leq 1$, then there exists $h \in(0,1)$ with $u(h t) \geq$ $v(t) \star u(t)$,

(F-3) if, for some constant $k \in(0,1)$, we have $F(u(k t), u(t), 1,1, u(t), u(t)) \geq$ 1 for any fixed $t>0$ and any nondecreasing function $u:(0, \infty) \rightarrow I$, then $u(k t) \geq u(t)$.

Now, let $\mathcal{F}$ be the set of all real continuous functions $F: I^{6} \rightarrow \mathbb{R}$ satisfying the conditions $(\mathrm{F}-1) \sim(\mathrm{F}-3)$.

Example 3. Let $F\left(u_{1}, \ldots, u_{6}\right)=\frac{u_{1}}{\min \left\{u_{2}, \ldots, u_{6}\right\}}$ and $a \star b=\min \{a, b\}$.

Let $t>0,0<u(t), v(t) \leq 1, k \in\left(0, \frac{1}{2}\right)$, where $u, v:[0, \infty) \rightarrow I$ are nondecreasing functions. Now, suppose that

i.e.,

$$
F\left(u(k t), v(t), v(t), u(t), 1, u\left(\frac{t}{2}\right) \star v\left(\frac{t}{2}\right)\right) \geq 1,
$$

$$
\begin{aligned}
F\left(u(k t), v(t), v(t), u(t), 1, u\left(\frac{t}{2}\right) \star v\left(\frac{t}{2}\right)\right) & =\frac{u(k t)}{\min \left\{v(t), u(t), 1, u\left(\frac{t}{2}\right) \star v\left(\frac{t}{2}\right)\right\}} \\
& =\frac{u(k t)}{\min \left\{v\left(\frac{t}{2}\right), u\left(\frac{t}{2}\right)\right\}} \geq 1 .
\end{aligned}
$$


Thus $u(h t) \geq v(t) \star u(t)$ if $h=2 k \in(0,1)$. A similar argument works if $\left(F_{b}\right)$ is assumed. Finally, suppose that $t>0$ is fixed, $u:(0, \infty) \rightarrow I$ is a nondecreasing function and

$$
F(u(k t), u(t), 1,1, u(t), u(t))=\frac{u(k t)}{u(t)} \geq 1
$$

for some $k \in(0,1)$. Then we have $u(k t) \geq u(t)$ and thus $F \in \mathcal{F}$.

Example 4. Let $F\left(u_{1}, \ldots, u_{6}\right)=\frac{u_{1} \max \left\{u_{2}, u_{3}, u_{4}\right\}}{\min \left\{u_{5}, u_{6}\right\}}$ and $\star$ be a continuous $t$-norm.

Let $t>0,0<u(t), v(t) \leq 1, k \in\left(0, \frac{1}{2}\right)$, where $u, v:[0, \infty) \rightarrow I$ are nondecreasing functions. Now, suppose that

$$
F\left(u(k t), v(t), v(t), u(t), 1, u\left(\frac{t}{2}\right) \star v\left(\frac{t}{2}\right)\right) \geq 1,
$$

i.e.,

$$
F\left(u(k t), v(t), v(t), u(t), 1, u\left(\frac{t}{2}\right) \star v\left(\frac{t}{2}\right)\right)=\frac{u(k t) \max \{v(t), u(t)\}}{u\left(\frac{t}{2}\right) \star v\left(\frac{t}{2}\right)} \geq 1 .
$$

Thus $u(h t) \geq v(t) \star u(t)$ if $h=2 k \in(0,1)$. A similar argument works if $\left(F_{b}\right)$ is assumed. Finally, suppose that $t>0$ is fixed, $u:(0, \infty) \rightarrow I$ is a nondecreasing function and

$$
F(u(k t), u(t), 1,1, u(t), u(t))=\frac{u(k t)}{u(t)} \geq 1
$$

for some $k \in(0,1)$. Then we have $u(k t) \geq u(t)$ and thus $F \in \mathcal{F}$.

Example 5. Let $F\left(u_{1}, \ldots, u_{6}\right)=\frac{\left(u_{1}\right)^{3}}{\left[u_{2} \star u_{3} \star u_{4}\right] \max \left\{u_{5}, u_{6}\right\}}$ and $a \star b=a b$.

Let $t>0,0<u(t), v(t) \leq 1, k \in(0,1)$, where $u, v:[0, \infty) \rightarrow I$ are nondecreasing functions. Now, suppose that

$$
F\left(u(k t), v(t), v(t), u(t), 1, u\left(\frac{t}{2}\right) \star v\left(\frac{t}{2}\right)\right) \geq 1,
$$

i.e.,

$$
F\left(u(k t), v(t), v(t), u(t), 1, u\left(\frac{t}{2}\right) \star v\left(\frac{t}{2}\right)\right)=\frac{(u(k t))^{3}}{(v(t))^{2} u(t)} \geq 1 .
$$

Thus we have

$$
u(k t)=u(h t) \geq(v(t))^{\frac{2}{3}}(u(t))^{\frac{1}{3}} \geq v(t) u(t)=v(t) \star u(t)
$$

if $h=k \in(0,1)$. A similar argument works if $\left(F_{b}\right)$ is assumed. Finally, suppose that $t>0$ is fixed, $u:[0, \infty) \rightarrow I$ is a nondecreasing function and

$$
F(u(k t), u(t), 1,1, u(t), u(t))=\frac{(u(k t))^{3}}{(u(t))^{2}} \geq 1
$$

for some $k \in(0,1)$. Then we have $u(k t) \geq u(t)$ and thus $F \in \mathcal{F}$. 


\section{Common fixed point theorems}

In this section, we prove some common fixed point theorems for four mappings on complete fuzzy metric spaces.

Theorem 1. Let $(X, M, \star)$ be a complete fuzzy metric space with $a \star b=$ $\min \{a, b\}$ for all $a, b \in I$ and $A, B, S$ and $T$ be mappings from $X$ into itself satisfying the conditions:

(i) $S(X) \subseteq B(X)$ and $T(X) \subseteq A(X)$,

(ii) one of the mappings $S, T, A, B$ is continuous,

(iii) $S$ and $A$ as well as $T$ and $B$ are compatible of type $(\alpha)$

(iv) there exist $k \in(0,1)$ and $F \in \mathcal{F}$ such that

$$
\begin{aligned}
& F(M(S x, T y, k t), M(A x, B y, t), M(S x, A x, t), \\
& M(T y, B y, t), M(S x, B y, t), M(T y, A x, t))
\end{aligned}
$$

for all $x, y \in X$ and $t>0$.

(v) for all $x, y \in X, M(x, y, t) \rightarrow 1$ as $t \rightarrow \infty$.

Then $A, B, S$ and $T$ have a unique common fixed point in $X$.

Proof. Let $x_{0}$ be an arbitrary point of $X$. From (i), we can construct a sequence $\left\{y_{n}\right\}$ in $X$ as follows:

$$
y_{2 n+1}=S x_{2 n}=B x_{2 n+1} \text { and } y_{2 n+2}=T x_{2 n+1}=A x_{2 n+2}
$$

for all $n=0,1, \ldots$. Then, by (iv), we have, for any $t>0$,

$$
\begin{aligned}
& F\left(M\left(S x_{2 n}, T x_{2 n+1}, k t\right), M\left(A x_{2 n}, B x_{2 n+1}, t\right), M\left(S x_{2 n}, A x_{2 n}, t\right),\right. \\
& \left.M\left(T x_{2 n+1}, B x_{2 n+1}, t\right), M\left(S x_{2 n}, B x_{2 n+1}, t\right), M\left(T x_{2 n+1}, A x_{2 n}, t\right)\right) \\
\geq & 1
\end{aligned}
$$

and so

$$
\begin{aligned}
& F\left(M\left(S x_{2 n}, T x_{2 n+1}, k t\right), M\left(T x_{2 n-1}, S x_{2 n}, t\right), M\left(S x_{2 n}, T x_{2 n-1}, t\right),\right. \\
& \left.\quad M\left(T x_{2 n+1}, S x_{2 n}, t\right), 1, M\left(T x_{2 n+1}, S x_{2 n}, \frac{t}{2}\right) \star M\left(S x_{2 n}, T x_{2 n-1}, \frac{t}{2}\right)\right)
\end{aligned}
$$$$
\geq 1 \text {. }
$$

By (F-2), we have

$$
M\left(S x_{2 n}, T x_{2 n+1}, h t\right) \geq M\left(S x_{2 n}, T x_{2 n-1}, t\right) \star M\left(S x_{2 n}, T x_{2 n+1}, t\right)
$$

and so

$$
M\left(y_{2 n+1}, y_{2 n+2}, h t\right) \geq M\left(y_{2 n+1}, y_{2 n}, t\right) \star M\left(y_{2 n+1}, y_{2 n+2}, t\right),
$$

which implies (note $a \star b=\min \{a, b\})$ that

$$
M\left(y_{2 n+1}, y_{2 n+2}, h t\right) \geq M\left(y_{2 n+1}, y_{2 n}, t\right)=M\left(y_{2 n}, y_{2 n+1}, t\right) .
$$

Again, by (F-2), we have

$$
M\left(y_{2 n+1}, y_{2 n}, h t\right) \geq M\left(y_{2 n}, y_{2 n-1}, t\right) .
$$


In general, we have, for all $m=1,2, \ldots$ and $t>0$,

$$
M\left(y_{m+1}, y_{m+2}, h t\right) \geq M\left(y_{m+1}, y_{m}, t\right)=M\left(y_{m}, y_{m+1}, t\right) .
$$

To prove that $\left\{y_{n}\right\}$ is a Cauchy sequence, first, we prove that, for any $0<$ $\lambda<1$ and $t>0$,

$$
M\left(y_{n+1}, y_{n+m+1}, t\right)>1-\lambda
$$

for all $n \geq n_{0}$ and $m \in \mathbb{N}$. Here we use induction. From (1), we have

$$
M\left(y_{n+1}, y_{n+2}, t\right) \geq M\left(y_{n}, y_{n+1}, \frac{t}{h}\right) \geq \cdots \geq M\left(y_{1}, y_{2}, \frac{t}{h^{n}}\right) \rightarrow 1
$$

as $n \rightarrow \infty$, i.e., for any $t>0$ and $\lambda \in(0,1)$, we can choose $n_{0} \in \mathbb{N}$ such that

$$
M\left(y_{n+1}, y_{n+2}, t\right)>1-\lambda .
$$

Thus (2) is true for $m=1$. Suppose that (2) is true for some $m \in \mathbb{N}$. Then we shall show that it is also true for $m+1 \in \mathbb{N}$. Using the definition of fuzzy metric space, (1) and (2) (which is true for $m \in \mathbb{N}$ ), we have

$$
M\left(y_{n+1}, y_{n+m+2}, t\right) \geq M\left(y_{n+1}, y_{n+m+1}, \frac{t}{2}\right) \star M\left(y_{n+m+1}, y_{n+m+2}, \frac{t}{2}\right) \geq 1-\lambda .
$$

Hence (2) is true for $m+1 \in \mathbb{N}$. Thus $\left\{y_{n}\right\}$ is Cauchy sequence in $X$. Since $(X, M, \star)$ is complete, $\left\{y_{n}\right\}$ converges to a point $z \in X$. Since $\left\{A x_{2 n+2}\right\}$, $\left\{B x_{2 n+1}\right\},\left\{S x_{2 n}\right\}$ and $\left\{T x_{2 n+1}\right\}$ are subsequences of $\left\{y_{n}\right\}$, we have $A x_{2 n+2}$, $B x_{2 n+1}, S x_{2 n}, T x_{2 n+1} \rightarrow z$ as $n \rightarrow \infty$.

Now, suppose that $A$ is continuous. Then the sequence $\left\{A S x_{2 n}\right\}$ converges to $A z$ as $n \rightarrow \infty$. Notice that, for any $t>0$,

$$
M\left(S A x_{2 n}, A z, t\right) \geq M\left(S A x_{2 n}, A A x_{2 n}, \frac{t}{2}\right) \star M\left(A A x_{2 n}, A z, \frac{t}{2}\right) .
$$

Now, since $A$ is continuous and $S, A$ are compatible of type $(\alpha)$, letting $n \rightarrow \infty$, we deduce that the sequence $\left\{S A x_{2 n}\right\}$ converges to $A z$ as $n \rightarrow \infty$. Using (iv), we have, for any $t>0$,

$$
\begin{aligned}
& F\left(M\left(S A x_{2 n}, T x_{2 n+1}, k t\right), M\left(A A x_{2 n}, B x_{2 n+1}, t\right), M\left(S A x_{2 n}, A A x_{2 n}, t\right),\right. \\
\geq & \left.M\left(T x_{2 n+1}, B x_{2 n+1}, t\right), M\left(S A x_{2 n}, B x_{2 n+1}, t\right), M\left(T x_{2 n+1}, A A x_{2 n}, t\right)\right)
\end{aligned}
$$

and then, by letting $n \rightarrow \infty$, since $F$ is continuous, we have

$$
F(M(A z, z, k t), M(A z, z, t), 1,1, M(A z, z, t), M(A z, z, t)) \geq 1 .
$$

Therefore, from (F-3), we have $M(A z, z, k t) \geq M(A z, z, t)$. From Lemma 2 , we have $A z=z$. Furthermore, by (iv), we have

$$
\begin{aligned}
& F\left(M\left(S z, T x_{2 n+1}, k t\right), M\left(A z, B x_{2 n+1}, t\right), M(A z, S z, t),\right. \\
& \left.M\left(T x_{2 n+1}, B x_{2 n+1}, t\right), M\left(S z, B x_{2 n+1}, t\right), M\left(T x_{2 n+1}, A z, t\right)\right) \\
\geq & 1 .
\end{aligned}
$$


and, letting $n \rightarrow \infty$, we get

$$
F(M(S z, z, k t), 1, M(S z, z, t), 1, M(S z, z, t), 1) \geq 1 .
$$

On the other hand, since

$$
M(S z, z, t) \geq M\left(S z, z, \frac{t}{2}\right)=M\left(S z, z, \frac{t}{2}\right) \star 1
$$

and $F$ is nonincreasing in the fifth variable, we have, for any $t>0$,

$$
\begin{aligned}
& F\left(M(S z, z, k t), 1, M(S z, z, t), 1, M\left(S z, z, \frac{t}{2}\right) \star 1,1\right) \\
\geq & F(M(S z, z, k t), 1, M(S z, z, t), 1, M(S z, z, t), 1) \\
\geq & 1,
\end{aligned}
$$

which implies, by (F-2), that $S z=z$. This means that $z$ is the range of $S$ and, since $S(X) \subseteq B(X)$, there exists a point $u \in X$ such that $B u=z$. Using (iv), we have successively

$$
\begin{aligned}
& F(M(S z, T u, k t), M(A z, B u, t), M(S z, A z, t), \\
&M(T u, B u, t), M(S z, B u, t), M(T u, A z, t)) \\
&= F(M(z, T u, k t), 1,1, M(z, T u, t), 1, M(z, T u, t)) \\
& \geq \quad 1,
\end{aligned}
$$

which implies, by (F-2), that $z=T u$. Since $B u=T u=z$ and $B, T$ are compatible of type $(\alpha)$, we have $T T u=B T u$ so $T z=T T u=B T u=B z$. Therefore, from (iv), we have, for any $t>0$,

$$
\begin{aligned}
& F(M(S z, T z, k t), M(A z, B z, t), M(S z, A z, t), \\
& M(T z, B z, t), M(S z, B z, t), M(T z, A z, t)) \\
= & F(M(z, T z, k t), M(z, T z, t), 1,1, M(z, T z, t), M(z, T z, t)) \\
\geq & 1 .
\end{aligned}
$$

Thus, from (F-3), we have $M(z, T z, k t) \geq M(z, T z, t)$. Again, from Lemma 2, we have $z=T z=B z$. Consequently, $z$ is a common fixed point of $S, T, A$ and $B$. The same result holds if we assume that $B$ is continuous instead of $A$.

Now, suppose that $S$ is continuous. Then the sequence $\left\{S A x_{2 n}\right\}$ converges to $S z$ as $n \rightarrow \infty$. Notice that, for any $t>0$,

$$
M\left(A S x_{2 n}, S z, t\right) \geq M\left(A S x_{2 n}, S S x_{2 n}, \frac{t}{2}\right) \star M\left(S S x_{2 n}, S z, \frac{t}{2}\right) .
$$

Now, since $S$ is continuous and $S, A$ are compatible of type $(\alpha)$, letting $n \rightarrow \infty$, we deduce that the sequence $\left\{A S x_{2 n}\right\}$ converges to $S z$. Using (iv), we have, for any $t>0$,

$$
\begin{array}{r}
F\left(M\left(S S x_{2 n}, T x_{2 n+1}, k t\right), M\left(A S x_{2 n}, B x_{2 n+1}, t\right), M\left(S S x_{2 n}, A S x_{2 n}, t\right),\right. \\
\\
\geq \quad 1
\end{array}
$$


and then, by letting $n \rightarrow \infty$, since $F$ is continuous, we have

$$
F(M(S z, z, k t), M(S z, z, t), 1,1, M(S z, z, t), M(S z, z, t)) \geq 1 .
$$

Thus, from (F-3), we have $M(S z, z, k t) \geq M(S z, z, t)$. Again, from Lemma 2, we have $S z=z$. This means that $z$ is the range of $S$ and, since $S(X) \subseteq B(X)$, there exists a point $v \in X$ such that $B v=z$. Using (iv), we have, for any $t>0$,

$$
\begin{aligned}
& F\left(M\left(S S x_{2 n}, T v, k t\right), M\left(A S x_{2 n}, B v, t\right), M\left(S S x_{2 n}, A S x_{2 n}, t\right),\right. \\
& \left.M(T v, B v, t), M\left(S S x_{2 n}, B v, t\right), M\left(T v, A S x_{2 n}, t\right)\right) \\
\geq & 1 .
\end{aligned}
$$

Letting $n \rightarrow \infty$, we get

$$
F(M(z, T v, k t), 1,1, M(z, T v, t), 1, M(z, T v, t)) \geq 1,
$$

which implies by (F-2), that $z=T v$. Since $B v=T v=z$ and $B, T$ are compatible of type $(\alpha)$, we have $T B v=B B v$ and so $T z=T B v=B B v=B z$. Thus, from (iv), we have

$$
\begin{aligned}
& F\left(M\left(S x_{2 n}, T z, k t\right), M\left(A x_{2 n}, B z, t\right), M\left(S x_{2 n}, A x_{2 n}, t\right),\right. \\
& \left.M(T z, B z, t), M\left(S x_{2 n}, B z, t\right), M\left(T z, A x_{2 n}, t\right)\right) \\
\geq & 1 .
\end{aligned}
$$

Letting $n \rightarrow \infty$, we get

$$
F(M(z, T z, k t), M(z, T z, t), 1,1, M(z, T z, t), M(z, T z, t)) \geq 1 .
$$

Thus $z=T z=B z$. This means that $z$ is the range of $T$ and, since $T(X) \subseteq$ $A(X)$, there exists $w \in X$ such that $A w=z$. Thus, from (iv), we have, for any $t>0$,

$$
\begin{aligned}
& F(M(S w, T z, k t), M(A w, B z, t), M(S w, A w, t), \\
& M(T z, B z, t), M(S w, B z, t), M(T z, A w, t)) \\
= & F(M(S w, z, k t), 1, M(S w, z, t), 1, M(S w, z, t), 1) \\
\geq & 1
\end{aligned}
$$

and, by (F-2), we have $z=S w=A w$. Since $S w=A w=z$ and $S, A$ are compatible of type $(\alpha)$, we have $z=S z=S A w=A A w=A z$ and thus $z=A z$. Consequently, $z$ is a common fixed point of $S, T, A$ and $B$. The same result holds if we assume that $T$ is continuous instead of $S$.

Finally, we show that the point $z$ is unique common fixed point of $S, T$, $A$ and $B$. Suppose that $S, T, A$ and $B$ have another common fixed point $z_{1}$. Then, by (iv), we have, for any $t>0$,

$$
\begin{aligned}
& F\left(M\left(S z, T z_{1}, k t\right), M\left(A z, B z_{1}, t\right), M(S z, A z, t)\right. \\
& M\left(T z_{1}, B z_{1}, t\right), M\left(S z, B z_{1}, t\right), M\left(T z_{1}, A z, t\right) \\
= & F\left(M\left(z, z_{1}, k t\right), M\left(z, z_{1}, t\right), 1,1, M\left(z, z_{1}, t\right), M\left(z, z_{1}, t\right)\right) \\
\geq & 1 .
\end{aligned}
$$


Thus, from (F-3), we have $M\left(z, z_{1}, k t\right) \geq M\left(z, z_{1}, t\right)$. From Lemma 2 , we have $z=z_{1}$. This completes the proof.

Now we give a fixed point theorem using compatible maps of type $(\beta)$.

Theorem 2. Let $(X, M, \star)$ be a complete fuzzy metric space with $a \star b=$ $\min \{a, b\}$ for all $a, b \in I$ and $A, B, S$ and $T$ be mappings from $X$ into itself satisfying the conditions:

(i) $S(X) \subseteq B(X)$ and $T(X) \subseteq A(X)$,

(ii) one of the mappings $S, T, A, B$ is continuous,

(iii) $S$ and $A$ as well as $T$ and $B$ are compatible of type $(\beta)$

(iv) there exist $k \in(0,1)$ and $F \in \mathcal{F}$ such that

$$
\begin{gathered}
\\
\quad F(M(S x, T y, k t), M(A x, B y, t), M(S x, A x, t), \\
\quad M(T y, B y, t), M(S x, B y, t), M(T y, A x, t))
\end{gathered}
$$

for all $x, y \in X$ and $t>0$.

(v) for all $x, y \in X, M(x, y, t) \rightarrow 1$ as $t \rightarrow \infty$.

Then $A, B, S$ and $T$ have a unique common fixed point in $X$.

Proof. We can show that $\left\{y_{n}\right\}$ is Cauchy sequence in $X$ as in Theorem 1 . Again, since $(X, M, \star)$ is complete, $\left\{y_{n}\right\}$ converges to a point $z \in X$. Since $\left\{A x_{2 n+2}\right\},\left\{B x_{2 n+1}\right\},\left\{S x_{2 n}\right\}$ and $\left\{T x_{2 n+1}\right\}$ are subsequences of $\left\{y_{n}\right\}$, we have $A x_{2 n+2}, B x_{2 n+1}, S x_{2 n}, T x_{2 n+1} \rightarrow z$ as $n \rightarrow \infty$.

Now, suppose that $A$ is continuous. Then the sequence $\left\{A S x_{2 n}\right\}$ converges to $A z$ as $n \rightarrow \infty$. From Proposition 4 , the sequence $\left\{S S x_{2 n}\right\}$ converges to $A z$ as $n \rightarrow \infty$. Now using (iv), we have, for any $t>0$,

$$
\begin{aligned}
& F\left(M\left(S S x_{2 n}, T x_{2 n+1}, k t\right), M\left(A S x_{2 n}, B x_{2 n+1}, t\right), M\left(S S x_{2 n}, A S x_{2 n}, t\right),\right. \\
& \left.M\left(T x_{2 n+1}, B x_{2 n+1}, t\right), M\left(S S x_{2 n}, B x_{2 n+1}, t\right), M\left(T x_{2 n+1}, A S x_{2 n}, t\right)\right) \\
\geq & 1 .
\end{aligned}
$$

and then, by letting $n \rightarrow \infty$, since $F$ is continuous, we have

$$
F(M(A z, z, k t), M(A z, z, t), 1,1, M(A z, z, t), M(A z, z, t)) \geq 1 .
$$

Therefore, from (F-3), we have $M(A z, z, k t) \geq M(A z, z, t)$. From Lemma 2, we have $A z=z$. Furthermore, by (iv), we have

$$
\begin{aligned}
& F\left(M\left(S z, T x_{2 n+1}, k t\right), M\left(A z, B x_{2 n+1}, t\right), M(A z, S z, t),\right. \\
& \left.M\left(T x_{2 n+1}, B x_{2 n+1}, t\right), M\left(S z, B x_{2 n+1}, t\right), M\left(T x_{2 n+1}, A z, t\right)\right) \\
\geq & 1 .
\end{aligned}
$$

and, letting $n \rightarrow \infty$, we get

$$
F(M(S z, z, k t), 1, M(S z, z, t), 1, M(S z, z, t), 1) \geq 1 .
$$


On the other hand, since

$$
M(S z, z, t) \geq M\left(S z, z, \frac{t}{2}\right)=M\left(S z, z, \frac{t}{2}\right) \star 1
$$

and $F$ is nonincreasing in the fifth variable, we have, for any $t>0$,

$$
\begin{aligned}
& F\left(M(S z, z, k t), 1, M(S z, z, t), 1, M\left(S z, z, \frac{t}{2}\right) \star 1,1\right) \\
\geq & F(M(S z, z, k t), 1, M(S z, z, t), 1, M(S z, z, t), 1) \\
\geq & 1,
\end{aligned}
$$

which implies, by (F-2), that $S z=z$. This means that $z$ is the range of $S$ and, since $S(X) \subseteq B(X)$, there exists a point $u \in X$ such that $B u=z$. Using (iv), we have successively

$$
\begin{aligned}
& F(M(S z, T u, k t), M(A z, B u, t), M(S z, A z, t), \\
&M(T u, B u, t), M(S z, B u, t), M(T u, A z, t)) \\
&= F(M(z, T u, k t), 1,1, M(z, T u, t), 1, M(z, T u, t)) \\
& \geq \quad 1,
\end{aligned}
$$

which implies, by (F-2), that $z=T u$. Since $B u=T u=z$ and $B, T$ are compatible of type $(\beta)$, we have $T T u=B B u$ so $T z=T T u=B B u=B z$. Therefore, from (iv), we have, for any $t>0$,

$$
\begin{aligned}
& F(M(S z, T z, k t), M(A z, B z, t), M(S z, A z, t) \\
& M(T z, B z, t), M(S z, B z, t), M(T z, A z, t)) \\
=\quad & F(M(z, T z, k t), M(z, T z, t), 1,1, M(z, T z, t), M(z, T z, t)) \\
\geq & 1 .
\end{aligned}
$$

Thus, from (F-3), we have $M(z, T z, k t) \geq M(z, T z, t)$. Again, from Lemma 2, we have $z=T z=B z$. Consequently, $z$ is a common fixed point of $S, T, A$ and $B$. The same result holds if we assume that $B$ is continuous instead of $A$.

Now, suppose that $S$ is continuous. Then the sequence $\left\{S A x_{2 n}\right\}$ converges to $S z$ as $n \rightarrow \infty$. From Proposition 4 , the sequence $\left\{A A x_{2 n}\right\}$ converges to $S z$ as $n \rightarrow \infty$. Now using (iv), we have, for any $t>0$,

$$
\begin{array}{r}
F\left(M\left(S A x_{2 n}, T x_{2 n+1}, k t\right), M\left(A A x_{2 n}, B x_{2 n+1}, t\right), M\left(S A x_{2 n}, A A x_{2 n}, t\right),\right. \\
\left.\geq \quad M\left(T x_{2 n+1}, B x_{2 n+1}, t\right), M\left(S A x_{2 n}, B x_{2 n+1}, t\right), M\left(T x_{2 n+1}, A A x_{2 n}, t\right)\right)
\end{array}
$$

and then, by letting $n \rightarrow \infty$, since $F$ is continuous, we have

$$
F(M(S z, z, k t), M(S z, z, t), 1,1, M(S z, z, t), M(S z, z, t)) \geq 1 .
$$

Thus, from (F-3), we have $M(S z, z, k t) \geq M(S z, z, t)$. Again, from Lemma 2, we have $S z=z$. This means that $z$ is the range of $S$ and, since $S(X) \subseteq B(X)$, 
there exists a point $v \in X$ such that $B v=z$. Using (iv), we have, for any $t>0$,

$$
\begin{aligned}
& F\left(M\left(S A x_{2 n}, T v, k t\right), M\left(A A x_{2 n}, B v, t\right), M\left(S A x_{2 n}, A A x_{2 n}, t\right),\right. \\
& \left.M(T v, B v, t), M\left(S A x_{2 n}, B v, t\right), M\left(T v, A A x_{2 n}, t\right)\right) \\
\geq & 1 .
\end{aligned}
$$

Letting $n \rightarrow \infty$, we get

$$
F(M(z, T v, k t), 1,1, M(z, T v, t), 1, M(z, T v, t)) \geq 1,
$$

which implies by $(\mathrm{F}-2)$, that $z=T v$. Since $B v=T v=z$ and $B, T$ are compatible of type $(\beta)$, we have $T T v=B B v$ and so $T z=T T v=B B v=B z$. Thus, from (iv), we have

$$
\begin{aligned}
& F\left(M\left(S x_{2 n}, T z, k t\right), M\left(A x_{2 n}, B z, t\right), M\left(S x_{2 n}, A x_{2 n}, t\right),\right. \\
& \left.M(T z, B z, t), M\left(S x_{2 n}, B z, t\right), M\left(T z, A x_{2 n}, t\right)\right)
\end{aligned}
$$

Letting $n \rightarrow \infty$, we get

$$
F(M(z, T z, k t), M(z, T z, t), 1,1, M(z, T z, t), M(z, T z, t)) \geq 1 .
$$

Thus $z=T z=B z$. This means that $z$ is the range of $T$ and, since $T(X) \subseteq$ $A(X)$, there exists $w \in X$ such that $A w=z$. Thus, from (iv), we have, for any $t>0$,

$$
\begin{aligned}
& F(M(S w, T z, k t), M(A w, B z, t), M(S w, A w, t), \\
& M(T z, B z, t), M(S w, B z, t), M(T z, A w, t)) \\
= & F(M(S w, z, k t), 1, M(S w, z, t), 1, M(S w, z, t), 1) \\
\geq & 1
\end{aligned}
$$

and, by (F-2), we have $z=S w=A w$. Since $S w=A w=z$ and $S, A$ are compatible of type $(\beta)$, we have $z=S z=S S w=A A w=A z$ and thus $z=A z$. Consequently, $z$ is a common fixed point of $S, T, A$ and $B$. The same result holds if we assume that $T$ is continuous instead of $S$.

Now, we give an example to illustrate our Theorems.

Example 6. Let $(X, M, \star)$ be a fuzzy metric space with $X=[0,1]$, a $t$-norm $\star$ be defined by $a \star b=\min \{a, b\}$ for all $a, b \in[0,1]$ and $M$ be a fuzzy set on $X^{2} \times(0, \infty)$ defined by

$$
M(x, y, t)=\left[\exp \left(\frac{|x-y|}{t}\right)\right]^{-1}
$$

for all $x, y \in X$ and $t>0$. Let $F: I^{6} \rightarrow \mathbb{R}$ be defined as in Example 3 and define the mappings $A, B, S, T: X \rightarrow X$ by

$$
A x=x, B x=\frac{x}{2}, S x=\frac{x}{4}, T x=\frac{x}{8},
$$


respectively. Then, for some $k \in\left[\frac{1}{2}, 1\right)$, we have

$$
\begin{aligned}
M(S x, T y, k t)= & {\left[\exp \left(\frac{\left|\frac{x}{8}-\frac{y}{4}\right|}{k t}\right)\right]^{-1} } \\
\geq & {\left[\exp \left(\frac{\left|\frac{x}{4}-\frac{y}{2}\right|}{t}\right)\right]^{-1} } \\
= & M(A x, B y, t) \\
\geq & \min \{M(A x, B y, t), M(S x, A x, t), M(T y, B y, t), \\
& M(S x, B y, t), M(T y, A x, t)\} .
\end{aligned}
$$

Thus the condition (iv) of Theorem 1 and 2 is satisfied. Also, it is obvious that the other conditions of the Theorems are satisfied and so zero is the unique fixed point of $A, B, S$ and $T$.

\section{References}

[1] R. Badard, Fixed point theorems for fuzzy numbers, Fuzzy Sets and Systems 13 (1984), 291-302.

[2] B. K. Bose and D. Sahani, Fuzzy mappings and fixed point theorems, Fuzzy Sets and Systems 21 (1987), 53-58.

[3] D. Butnariu, Fixed points for fuzzy mappings, Fuzzy Sets and Systems 7 (1982), 191207.

[4] S. S. Chang, Fixed point theorems for fuzzy mappings, Fuzzy Sets and Systems 17 (1985), 181-187.

[5] S. S. Chang, Y. J. Cho, B. S. Lee, J. S. Jung, and S. M. Kang, Coincidence point and minimization theorems in fuzzy metric spaces, Fuzzy Sets and Systems 88 (1997), $119-128$.

[6] S. S. Chang, Y. J. Cho, B. E. Lee, and G. M. Lee, Fixed degree and fixed point theorems for fuzzy mappings, Fuzzy Sets and Systems 87 (1997), 325-334.

[7] Y. J. Cho, Fixed points in fuzzy metric spaces, J. Fuzzy Math. 5 (1997), 949-962.

[8] Y. J. Cho, H. K. Pathak, S. M. Kang, and J. S. Jung, Common fixed points of compatible maps of type $(\beta)$ on fuzzy metric spaces, Fuzzy Sets and Systems 93 (1998), 99-111.

[9] J. X. Fang, On fixed point theorems in fuzzy metric spaces, Fuzzy Sets and Systems 46 (1992), 107-113

[10] A. George and P. Veeramani, On some results in fuzzy metric spaces, Fuzzy Sets and Systems 64 (1994), 395-399.

[11] M. Grabiec, Fixed points in fuzzy metric spaces, Fuzzy Sets and Systems 27 (1988), $385-389$.

[12] O. Hadzic, Fixed point theorems for multi-valued mappings in some classes of fuzzy metric spaces, Fuzzy Sets and Systems 29 (1989), 115-125.

[13] M. Imdad, S. Kumar, and M. S. Khan, Remarks on some fixed point theorems satisfying implicit relations, Rad. Math. 11 (2002), 135-143.

[14] J. S. Jung, Y. J. Cho, and J. K. Kim, Minimization theorems for fixed point theorems in fuzzy metric spaces and applications, Fuzzy Sets and Systems 61 (1994), 199-207.

[15] J. S. Jung, Y. J. Cho, S. S. Chang, and S. M. Kang, Coincidence theorems for setvalued mappings and Ekland's variational principle in fuzzy metric spaces, Fuzzy Sets and Systems 79 (1996), 239-250.

[16] S. N. Mishra, S. N. Sharma, and S. L. Singh, Common fixed points of maps in fuzzy metric spaces, Internat. J. Math. Math. Sci. 17 (1994), 253-258. 
ISHAK ALTUN AND DURAN TURKOGLU

[17] V. Popa, A general coincidence theorem for compatible multivalued mappings satisfying an implicit relation, Demonsratio Math. 33 (2000), 159-164.

[18] _ Some fixed point theorems for compatible mappings satisfying an implicit relation, Demonsratio Math. 32 (1999), 157-163.

[19] V. Popa and D. Turkoglu, Some fixed point theorems for hybrid contractions satisfying an implicit relation, Stud. Cercet. Stint. Ser. Math. Univ. Bacau 8 (1998), 75-86.

[20] B. Schweizer and A. Sklar, Statistical metric spaces, Pacific J. Math. 10 (1960), 313-334.

[21] S. Sharma, Common fixed point theorems in fuzzy metric spaces, Fuzzy Sets and Systems 127 (2002), 345-352.

[22] S. Sharma and B. Desphande, Common fixed points of compatible maps of type $(\beta)$ on fuzzy metric spaces, Demonsratio Math. 35 (2002), 165-174.

[23] _ On compatible mappings satisfying an implicit relation in common fixed point consideration, Tamkang J. Math. 33 (2002), 245-252.

[24] B. Singh and M. S. Chauhan, Common fixed points of compatible maps in fuzzy metric spaces, Fuzzy Sets and Systems 115 (2000), 471-475.

[25] L. A. Zadeh, Fuzzy sets, Inform and Control 8 (1965), 338-353.

IShak Altun

Department of Mathematics

Faculty of Science And Arts

KIRKKALE UNIVERSITY

71450-Yahsihan, Kirikkale, Turkey

E-mail address: ialtun@kku.edu.tr, ishakaltun@yahoo.com

Duran Turkoglu

Department of Mathematics

Faculty of Science AND ARts

GAZI UNIVERSITY

06500-Teknikokullar, Ankara, Turkey

E-mail address: dturkoglu@gazi.edu.tr 